This item was submitted to Loughborough's Research Repository by the author.

Items in Figshare are protected by copyright, with all rights reserved, unless otherwise indicated.

\title{
Propagation and interaction of gravitational waves in some expanding backgrounds
}

PLEASE CITE THE PUBLISHED VERSION

PUBLISHER

(C) American Physical Society

LICENCE

CC BY-NC-ND 4.0

REPOSITORY RECORD

Alekseev, G.A., and J.B. Griffiths. 2019. "Propagation and Interaction of Gravitational Waves in Some Expanding Backgrounds". figshare. https://hdl.handle.net/2134/1716. 


\title{
Propagation and interaction of gravitational waves in some expanding backgrounds
}

\author{
Georgii A. Alekseev \\ Steklov Mathematical Institute, Vavilova 42, Moscow 117966, GSP-1, Moscow, Russia \\ Jerry B. Griffiths \\ Department of Mathematical Sciences, University of Technology, \\ Loughborough, Leicestershire LE11 3TU, United Kingdom
}

(Received 31 May 1995)

\begin{abstract}
An explicit class of solutions is given which represents a family of expanding gravitational waves with distinct wave fronts propagating into a variety of backgrounds, including vacuum Kasner and Friedmann-Robertson-Walker fluid models with a stiff equation of state. Complete global solutions are given, and it is indicated how solutions describing the collision of such waves may be constructed. Since the solutions obtained are regular apart from the initial singularity, it is concluded that this class of colliding gravitational waves do not introduce singularities into the space-time.
\end{abstract}

PACS number(s): 04.40.-b, 04.30.Nk

\section{INTRODUCTION}

The class of nonexpanding plane gravitational waves, which propagate in a flat background, are well known. In recent years considerable attention has been paid to the problem of the collision and interaction of such waves (for a review see [1]). The purpose of the present paper is to present a class of exact solutions that represents the propagation of expanding plane fronted gravitational waves in certain physically significant backgrounds and to consider solutions describing their collision and subsequent interaction.

Our attention will be restricted to space-times that contain two commuting, hypersurface orthogonal spacelike Killing vectors. For this, the line element can be written in terms of two null coordinates $u$ and $v$ in the Einstein-Rosen form

$$
d s^{2}=2 e^{-M} d u d v-e^{-U}\left(e^{V} d x^{2}+e^{-V} d y^{2}\right),
$$

where $U, V$, and $M$ are functions of $u$ and $v$ only. This is sufficient to describe both a suitable class of backgrounds and regions containing gravitational waves which will be identified by appropriate components of the Weyl tensor.

The space-times considered here admit a family of planes spanned by the two Killing vectors $\partial_{x}$ and $\partial_{y}$. These will form wave fronts of a gravitational wave, which will propagate into a background along a null hypersurface, normally given by $u=0$. The background region will be that for which $u<0$, while the region $u \geq 0$ will contain the gravitational wave.

The background will admit full plane symmetry if $V=0$ in that region. It will then either be conformally flat or of algebraic type $D$. However, we will also consider various backgrounds for which $V \neq 0$. These include certain cases that may be interpreted as having cylindrical symmetry.

The simplest expanding backgrounds to be considered are the vacuum Kasner space-times. These can easily be extended to include the presence of a stiff perfect fluid or a massless scalar field. Of particular interest here are the conformally flat Friedmann-RobertsonWalker (FRW) models containing a stiff fluid, particularly the spatially flat and open cases, which admit full plane symmetry. Some appropriate backgrounds will be described in Sec. III. In general, such backgrounds are often referred to as Gowdy universes.

The propagation and interaction of "gravitational solitons" in such backgrounds are well known following the work of Belinskii and Zakharov [2] and Belinskii [3]. Recent work on this topic has been thoroughly reviewed by Verdaguer [4]. Other inhomogeneous cosmological models containing gravitational and scalar waves have been previously reviewed by Carmeli, Charach, and Malin [5]. In this paper, however, attention is restricted to waves having well-defined wave fronts.

The collision and interaction of gravitational waves in a vacuum Kasner background was initially considered by Centrella and Matzner [6]. In a series of recent papers, the propagation and interaction of gravitational waves into spatially flat [7], [8], open [9], and closed [10] FRW stiff fluid backgrounds have also been described. In all these cases, however, the gravitational waves have been described in terms of the metric functions $U$ and $V$, but explicit expressions for $M$ have not been obtained. Nevertheless, it has been shown that the gravitational wave represented by these solutions is necessarily partially backscattered as it propagates.

In Sec. IV we will present complete solutions, including explicit expressions for $M$, for a general class of gravitational waves with well-defined wave fronts. Solutions describing the head-on collision of such waves and their subsequent interaction will be presented in Sec. V. There it will be shown that the components representing the gravitational waves are regular throughout the space-time. It is therefore concluded that the collision of initially ex- 
panding gravitational waves does not introduce future singularities. This is in marked contrast to the case of nonexpanding gravitational wave interactions in a flat background.

\section{FIELD EQUATIONS}

For space-times with the metric given by (1.1), the vacuum field equations take the form

$$
\begin{aligned}
U_{u v} & =U_{u} U_{v} \\
2 U_{v v} & =U_{v}^{2}+V_{v}^{2}-2 U_{v} M_{v} \\
2 U_{u u} & =U_{u}^{2}+V_{u}^{2}-2 U_{u} M_{u} \\
2 M_{u v} & =-U_{u} U_{v}+V_{u} V_{v} \\
2 V_{u v} & =U_{u} V_{v}+U_{v} V_{u}
\end{aligned}
$$

and the nonzero components of the Weyl tensor can be expressed in scale-invariant form as

$$
\begin{aligned}
& \Psi_{0}^{\circ}=-\frac{1}{2}\left(V_{v v}-U_{v} V_{v}+M_{v} V_{v}\right), \\
& \Psi_{2}^{\circ}=-\frac{1}{4}\left(U_{u} U_{v}-V_{u} V_{v}\right), \\
& \Psi_{4}^{\circ}=-\frac{1}{2}\left(V_{u u}-U_{u} V_{u}+M_{u} V_{u}\right) .
\end{aligned}
$$

It therefore follows that the gravitational waves will largely be determined by the metric function $V(u, v)$.

Equation (2.1) can immediately be integrated to give

$$
e^{-U}=f(u)+g(v),
$$

where $f(u)$ and $g(v)$ are arbitrary functions. It is often possible to adopt $f$ and $g$ as coordinates in place of $u$ and $v$. However, in order to include a wide range of backgrounds, it is convenient here generally to retain the functions $f(u)$ and $g(v)$.

It may also be noticed that Eqs. (2.1), (2.4), and (2.5) are the integrability conditions for Eqs. (2.2) and (2.3). Thus, if Eq. (2.5) is satisfied using (2.7), there automatically exists a function $M$ satisfying equations (2.2), (2.3), and (2.4). In principle, $M$ can then be obtained by quadrature, although not usually in closed form.

In terms of the functions $f$ and $g$, the main field equation (2.5) can be written as the Euler-Poisson-Darboux equation with noninteger coefficients

$$
(f+g) V_{f g}+\frac{1}{2} V_{f}+\frac{1}{2} V_{g}=0 .
$$

For any particular solution of this equation, the remaining function $M$ can then be found by putting

$$
e^{-M}=\frac{f^{\prime} g^{\prime}}{\sqrt{f+g}} e^{-S}
$$

where, from (2.2) and (2.3), $S$ must satisfy the subsidiary equations

$$
S_{f}=-\frac{1}{2}(f+g) V_{f}^{2}, \quad S_{g}=-\frac{1}{2}(f+g) V_{g}^{2},
$$

which are automatically integrable in view of (2.8).

We wish to consider a gravitational wave having a wave front given by $f=0$ (usually also $u=0$ ). This will be defined in the region $f \geq 0$ and will propagate into the background region $f<0$ as illustrated in Fig. 1. However, it is clear from (2.7) that a singularity occurs when $f+g=0$. This will generally be a curvature singularity corresponding to a kind of big bang from which the background emerges.

It is well known that, for any vacuum space-time with two commuting, orthogonally transitive spacelike Killing vectors, a solution containing a perfect fluid with a stiff equation of state $p=\rho$ can be generated using the method of Wainwright, Ince, and Marshman [11]. In the case when the Killing vectors are orthogonal, the procedure can be summarized as follows. Given any vacuum solution with the line element (1.1) with metric functions $U=-\ln [f(u)+g(v)], V$ and $M$, and any potential function $\sigma(f, g)$ satisfying the equation

$$
(f+g) \sigma_{f g}+\frac{1}{2} \sigma_{f}+\frac{1}{2} \sigma_{g}=0,
$$

a new stiff fluid solution is given by $U, V$, and $M+\Omega$, where the function $\Omega(u, v)$ is found by integrating the equations

$$
\Omega_{f}=-\frac{1}{2}(f+g){\sigma_{f}}^{2}, \quad \Omega_{g}=-\frac{1}{2}(f+g){\sigma_{g}}^{2} .
$$

The function $\sigma(f, g)$ may be considered either as a massless scalar field or as a potential for a stiff perfect fluid [12] with

$$
16 \pi \rho=e^{M+\Omega} \sigma_{u} \sigma_{v}=\sqrt{f+g} e^{S+\Omega} \sigma_{f} \sigma_{g} .
$$

It may also be noted that the Weyl tensor component $\Psi_{2}$ is modified to the form

$$
\Psi_{2}=-\frac{1}{12} e^{M+\Omega}\left(3 U_{u} U_{v}-3 V_{u} V_{v}-\sigma_{u} \sigma_{v}\right)
$$

It may be noticed that Eqs. (2.8) and (2.9) are formally identical to Eqs. (2.10) and (2.11). Thus any solutions of (2.8) and (2.9) representing gravitational waves may also be considered as possible solutions of (2.10) and (2.11) representing scalar waves or acoustic waves in a stiff perfect fluid. Although this dual interpretation is possible, the following sections will be written only in terms of gravitational waves in particular backgrounds.

In this approach it is, therefore, only necessary to construct solutions for gravitational waves in expanding vacuum backgrounds. To any such solution a scalar

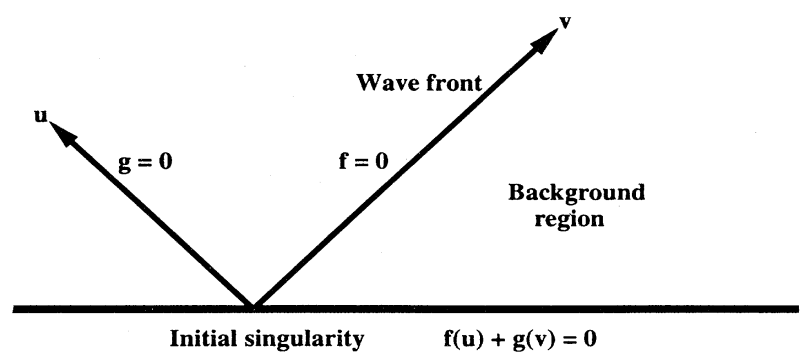

FIG. 1. A gravitational wave with wave front $f=0$ propagates into a background region, which contains an initial singularity at $f+g=0$. 
field (possibly including scalar waves) or stiff fluid (with or without independent acoustic waves) can always be added subsequently.

It is convenient here also to introduce alternative timelike and spacelike coordinates $t$ and $z$ defined by

$$
t=f+g, \quad z=g-f .
$$

In this case, the line element (1.1) can be rewritten in the form

$$
d s^{2}=\frac{e^{-S}}{2 \sqrt{t}}\left(d t^{2}-d z^{2}\right)-t\left(e^{V} d x^{2}+e^{-V} d y^{2}\right)
$$

the main vacuum field equation (2.8) becomes

$$
V_{t t}+\frac{1}{t} V_{t}-V_{z z}=0
$$

and the subsidiary equations $(2.9)$ become

$$
S_{t}=-\frac{1}{2} t\left(V_{t}^{2}+V_{z}^{2}\right), \quad S_{z}=-t V_{t} V_{z} .
$$

\section{POSSIBLE BACKGROUNDS}

The basic background that we wish to consider is that of a vacuum Kasner solution given here by

$$
V=a \ln (f+g), \quad S=-\frac{1}{2} a^{2} \ln (f+g),
$$

or $e^{V}=t^{a}$ and $e^{-S}=t^{a^{2} / 2}$, where $a$ is an arbitrary constant. By putting $\tilde{t}=t^{\left(a^{2}+3\right) / 4}$ and rescaling the coordinates, this may be expressed in the more familiar form of the Kasner metric

$$
d s^{2}=d \tilde{t}^{2}-\tilde{t}^{2 p_{1}} d x^{2}-\tilde{t}^{2 p_{2}} d y^{2}-\tilde{t}^{2 p_{3}} d z^{2}
$$

where the exponents are given by

$$
p_{1}=\frac{2(1-a)}{a^{2}+3}, \quad p_{2}=\frac{2(1+a)}{a^{2}+3}, \quad p_{3}=\frac{a^{2}-1}{a^{2}+3} .
$$

This space-time is flat when $a= \pm 1$. The use of this particular case enables us to consider expanding gravitational waves in a Minkowski background. In this case, however, there is a coordinate singularity instead of a curvature singularity when $t=0$. The above vacuum Kasner solution is of algebraic type $D$ when $a=0$.

To this family of backgrounds can always be added a scalar field or stiff fluid using the Wainwright-InceMarshman algorithm (2.10) and (2.11). One such solution is given by

$$
\sigma=b \ln (f+g), \quad \Omega=-\frac{1}{2} b^{2} \ln (f+g) .
$$

In this case, the line element is

$$
\begin{aligned}
d s^{2}=2(f+g)^{\left(a^{2}+b^{2}-1\right) / 2} d f d g & \\
& -(f+g)^{1+a} d x^{2}-(f+g)^{1-a} d y^{2} .
\end{aligned}
$$

This includes the spatially flat FRW stiff fluid model when $a=0$ and $b=\sqrt{3}$.
Another solution that is of particular physical interest is given by

$$
\begin{aligned}
& \sigma=b \ln \left(\frac{\sqrt{1+g}-\sqrt{1-f}}{\sqrt{1+g}+\sqrt{1-f}}\right), \\
& \Omega=\frac{b^{2}}{2} \ln \left(\frac{(1+g)(1-f)}{f+g}\right) .
\end{aligned}
$$

When $a=0$ and $b=\sqrt{3}$, this is the open FRW stiff fluid model. This can be seen by putting $f(u)=1-e^{-4 u}$ and $g(v)=e^{4 v}-1$, so that $\sigma=\sqrt{3} \ln \tanh (u+v)$. It is then in the form described by Bičák and Griffiths [9] in which the hypersurface $f=1$ is part of future null infinity.

The two FRW stiff fluid models included above are particularly appropriate to consider as backgrounds to gravitational waves, since they are conformally flat with $V(f, g)=0$. The gravitational waves can then be clearly identified in terms of the nonzero components of the Weyl tensor. All that is required is to find a solution of Eq. (2.8) for which $V(0, g)=0$ and $V_{f}(0, g)$ is bounded on a wave front given by $f=0$. In any more general background, a solution of (2.8) for $V(f, g)$, satisfying $V(0, g)=0$ and $V_{f}(0, g)$ bounded, can always be added to the background $V$, since (2.8) is linear. However, the properties of the gravitational wave added in this way are more difficult to identify.

In terms of the metric

$$
d s^{2}=e^{-M}\left(d \eta^{2}-d \tilde{z}^{2}\right)-e^{-U}\left(e^{V} d x^{2}+e^{-V} d y^{2}\right)
$$

in which we have now put $\eta=1 / \sqrt{2}(u+v)$ and $\tilde{z}=$ $1 / \sqrt{2}(u-v)$, the above two FRW models may be summarized in the form

$$
\begin{array}{lll}
k=0: & e^{-U}=2 \eta, & e^{-M}=\gamma 2 \eta, \\
& V=0, & \sigma=\sqrt{3} \ln \eta, \\
k=-1: & e^{-U}=\sinh 2 \eta e^{-2 \tilde{z}}, & e^{-M}=\gamma \sinh 2 \eta, \\
& V=0, & \sigma=\sqrt{3} \ln \tanh \eta
\end{array}
$$

where $\gamma$ is a constant related to the energy density of the fluid and some coordinates have been rescaled. In these cases, the initial big bang singularity occurs when $\eta=0$ and the surfaces of plane symmetry are clearly identified.

It is well known [13], however, that all the FRW spacetimes with a stiff fluid source may also be written in a different form using the same line element as that given above. The various cases may be summarized as

$$
\begin{array}{lll}
k=+1: & e^{-U}=\sin 2 \eta \sin 2 \tilde{z}, & e^{-M}=\gamma \sin 2 \eta \\
& e^{V}=\tan \tilde{z}, & \sigma=\sqrt{3} \ln \tan \eta \\
k=0: & e^{-U}=2 \eta \tilde{z}, & e^{-M}=\gamma 2 \eta \\
& e^{V}=\tilde{z}, & \sigma=\sqrt{3} \ln \eta \\
k=-1: & e^{-U}=\sinh 2 \eta \sinh 2 \tilde{z}, & e^{-M}=\gamma \sinh 2 \eta \\
& e^{V}=\tanh \tilde{z}, & \sigma=\sqrt{3} \ln \tanh \eta
\end{array}
$$

Expressed in the above notation, these forms of the FRW models may be defined by 


$$
\begin{aligned}
& k=+1: \quad f=\sin ^{2} u, \quad g=-\sin ^{2} v, \\
& V=\frac{1}{2} \ln \left(\frac{1-\sqrt{f} \sqrt{-g}-\sqrt{1-f} \sqrt{1+g}}{1+\sqrt{f} \sqrt{-g}+\sqrt{1-f} \sqrt{1+g}}\right), \\
& \sigma=\frac{\sqrt{3}}{2} \ln \left(\frac{1+\sqrt{f} \sqrt{-g}-\sqrt{1-f} \sqrt{1+g}}{1-\sqrt{f} \sqrt{-g}+\sqrt{1-f} \sqrt{1+g}}\right) \text {, } \\
& k=0: \quad f=u^{2}, \quad g=-v^{2} \text {, } \\
& V=-\ln (\sqrt{f}-\sqrt{-g}) \text {, } \\
& \sigma=\sqrt{3} \ln (\sqrt{f}+\sqrt{-g}), \\
& k=-1: \quad f=\sinh ^{2} u, \quad g=-\sinh ^{2} v, \\
& V=\frac{1}{2} \ln \left(\frac{\sqrt{1+f} \sqrt{1-g}-\sqrt{f} \sqrt{-g}-1}{\sqrt{1+f} \sqrt{1-g}-\sqrt{f} \sqrt{-g}+1}\right), \\
& \sigma=\frac{\sqrt{3}}{2} \ln \left(\frac{\sqrt{1+f} \sqrt{1-g}+\sqrt{f} \sqrt{-g}-1}{\sqrt{1+f} \sqrt{1-g}+\sqrt{f} \sqrt{-g}+1}\right) \text {. }
\end{aligned}
$$

In this notation, it is possible to consider FRW stiff fluid backgrounds of all three types. However, the solutions in this form identify different families of null hypersurfaces to those considered above. If $x$ is considered as a periodic (angular) coordinate, then $\tilde{z} \geq 0$ may be interpreted as the radial-like coordinate of a family of cylindrical surfaces. Solutions in these forms may, therefore, be taken as stiff fluid backgrounds to families of cylindrical gravitational waves.

It is also possible to consider background space-times having any multiple of the above expressions for $V$. Indeed, any solution of (2.8) may be taken in the background region and extended through the wave front. Such backgrounds will be included in the general class of Gowdy cosmological models. However, we will explicitly give complete solutions here only for the simplest backgrounds, namely, the vacuum Kasner and the spatially flat and open FRW stiff fluid models.

\section{EXPLICIT SOLUTIONS}

Our first task is to find a solution of Eq. (2.8) for which $V=0$ and $V_{f}$ is bounded on a wave front given by $f=0$. However, it is convenient here to start with this equation in the form (2.12) involving the coordinates $t=f+g$ and $z=g-f$. It may be observed that (2.12) admits a similarity solution. We may therefore consider solutions of the form

$$
V(t, z)=t^{n} H_{n}\left(\frac{z}{t}\right) .
$$

Substituting (4.1) into (2.12) gives the following equation for $H_{n}(\zeta)$ :

$$
\left(1-\zeta^{2}\right) H_{n}^{\prime \prime}+(2 n-1) \zeta H_{n}^{\prime}-n^{2} H_{n}=0,
$$

where

$$
\zeta=\frac{z}{t}=\frac{g-f}{g+f} \quad \text { and } \quad-1 \leq \zeta \leq 1 .
$$

It may be noted that $\zeta$ is a decreasing coordinate behind the wave front on which $\zeta=1$.
The substitution $\xi=\frac{1}{2}(1-\zeta)$ transforms Eq. (4.2) into the usual form of the hypergeometric equation, whose solution can be expressed in terms of hypergeometric functions in the form

$$
\begin{aligned}
V(t, \zeta)= & A_{n} t^{n} F\left(-n,-n ; \frac{1}{2}-n ; \frac{1}{2}(1-\zeta)\right) \\
& +B_{n} t^{n}(1-\zeta)^{n+\frac{1}{2}} F\left(\frac{1}{2}, \frac{1}{2} ; n+\frac{3}{2} ; \frac{1}{2}(1-\zeta)\right) .
\end{aligned}
$$

These solutions are valid for any arbitrary $n$, not necessarily an integer. The first of these sets of terms may be discarded, since they are not zero on the hypersurface $f=0$ on which $\zeta=1$. The second set, however, is zero on $f=0$ for $n>-\frac{1}{2}$. It is these solutions that have formed the basis of the previous papers [7-10].

In passing it may be observed that, in these solutions, the Weyl tensor components $\Psi_{4}, \Psi_{0}$, and $\Psi_{2}$ are necessarily nonzero. It follows that the gravitational wave that has been introduced with the defined wave front is necessarily backscattered as it propagates, and the space-time is algebraically general.

It has been found convenient here to continue to express the solutions in the form (4.1). This form, together with Eq. (4.2), yields an explicit set of solutions with some very nice properties. This particular form also enables complete integrals of the subsidiary equations (2.13) to be obtained.

It may first be noted that, for $n=0$, Eq. (4.2) admits the explicit solution

$$
H_{0}(\zeta)=\frac{1}{\pi} \arccos \zeta,
$$

which satisfies the condition $H_{0}(1)=0$. However, although this is zero on $f=0$, its derivative $V_{f}$ is unbounded on $f=0$. On its own, it therefore does not satisfy the necessary junction conditions across the gravitational wave front.

Secondly, by differentiating Eq. (4.2), it may be observed that, for any $n$, a particular solution $H_{n}(\zeta)$ satisfying the condition $H_{n}(1)=0$ can be calculated recursively from $H_{n-1}(\zeta)$ using the relation

$$
H_{n}(\zeta)=\int_{1}^{\zeta} H_{n-1}\left(\zeta^{\prime}\right) d \zeta^{\prime} .
$$

Starting with the above solution $H_{0}(\zeta)$ for $n=0$, a sequence of explicit solutions for $n=1,2, \ldots$ can now easily be constructed. The derivatives of the $n$th solution will satisfy the conditions

$$
H_{n}(1)=0, \quad H_{n}^{\prime}(1)=0, \quad \cdots, \quad H_{n}^{(n)}(1)=0
$$

while the $(n+1)$ th derivative will contain some integrable singularity (proportional to $1 / \sqrt{1-\zeta^{2}}$ ). It is convenient to rescale these solutions by multiplying by the numerical coefficient $1 / H_{n}(-1)$, so that the redefined solutions satisfy the conditions $H_{n}(-1)=1$. The solutions (18) then satisfy the boundary conditions

$$
V_{n}(f, g)=\left\{\begin{array}{cc}
0, & f=0 \\
f^{n}, & g=0
\end{array} \quad \text { for } \quad n=0,1,2, \ldots\right.
$$


By explicit calculation, it is found that the first few solutions in this series are

$$
\begin{aligned}
H_{0}(\zeta)= & \frac{1}{\pi} \arccos \zeta \\
H_{1}(\zeta)= & -\frac{1}{\pi} \zeta \arccos \zeta+\frac{1}{\pi} \sqrt{1-\zeta^{2}} \\
H_{2}(\zeta)= & \frac{1}{3 \pi}\left(1+2 \zeta^{2}\right) \arccos \zeta-\frac{1}{\pi} \zeta \sqrt{1-\zeta^{2}} \\
H_{3}(\zeta)= & -\frac{1}{5 \pi} \zeta\left(3+2 \zeta^{2}\right) \arccos \zeta \\
& +\frac{1}{15 \pi}\left(4+11 \zeta^{2}\right) \sqrt{1-\zeta^{2}}
\end{aligned}
$$

A particularly useful property of these solutions is that they enable complete integrals of the subsidiary equations (2.9) or (2.13) to be obtained. However, it is first convenient to rewrite these equations in the form

$$
\begin{aligned}
& S_{t}=-\frac{t}{2} V_{t}^{2}-\frac{1}{2 t}\left(1-\zeta^{2}\right) V_{\zeta}^{2}, \\
& S_{\zeta}=-t V_{t} V_{\zeta}+\zeta V_{\zeta}^{2}
\end{aligned}
$$

For any particular solution (4.1) given by $V=t^{n} H_{n}(\zeta)$ for any $n \neq 0$, these equations may be integrated to give

$$
S=-\frac{t^{2 n}}{4 n}\left[n^{2} H_{n}^{2}+\left(1-\zeta^{2}\right) H_{n}^{\prime 2}\right]
$$

It may now be noted that the above solutions only satisfy the boundary conditions for a propagating gravitational wave when $n \geq \frac{1}{2}$. Thus $V_{0}=a_{0} H_{0}(\zeta)$ does not satisfy the boundary conditions, but it can still be used to generate the above series $V_{n}$ for integer $n \geq 1$. It is then possible to consider a class of solutions of the form

$$
V=\sum_{n=1}^{\infty} a_{n} t^{n} H_{n}(\zeta)
$$

in which $a_{n}$ are arbitrary constants. It can be seen that solutions of this type correspond to a special case of the series expansions given previously where the expansion was in arbitrary powers of $f$ with coefficients that include hypergeometric functions of $-f / g$. In this case, the solution (4.5) corresponds to an expansion in half-integer powers of $f$, with the initial term of order $f^{3 / 2}$.

One advantage of the expansion given here is that it can be expressed explicitly in terms of more familiar functions. However, the main advantage is that, since it is expressed as a power series in $t$, the subsidiary equations in the form (4.4) can be integrated completely. When the background is taken to be a spatially flat or open FRW stiff fluid background or a type- $D$ Kasner background, then $V=0$ for $f<0$. For these cases, with a gravitational wave defined by (4.5) for $f \geq 0$, it can be shown that the explicit expression for $S$ for $f \geq 0$ is given by

$$
S=-\sum_{n=2}^{\infty} \frac{1}{2 n} t^{n} K_{n}
$$

where

$$
\begin{aligned}
K_{n}(\zeta)= & \sum_{k=1}^{n-1} a_{k} a_{n-k}\left[k(n-k) H_{k} H_{n-k}\right. \\
& \left.+\left(1-\zeta^{2}\right) H_{k}^{\prime} H_{n-k}^{\prime}\right]
\end{aligned}
$$

In the case of a gravitational wave propagating in a general vacuum Kasner background we may take, for $f \geq 0$,

$$
V=a_{0} \ln t+\sum_{n=1}^{\infty} a_{n} t^{n} H_{n}(\zeta)
$$

In this case the subsidiary equations may be integrated to give

$$
S=-\frac{a_{0}^{2}}{2} \ln t-a_{0} \sum_{n=1}^{\infty} a_{n} t^{n} H_{n}-\sum_{n=2}^{\infty} \frac{1}{2 n} t^{n} K_{n},
$$

using the same expression as above for $K_{n}(\zeta)$. To this solution, any scalar field or stiff perfect fluid may be added using the Wainwright-Ince-Marshman algorithm.

The class of explicit solutions described above are strictly valid only in the region defined by $-1 \leq \zeta \leq 1$ for which $f \geq 0$ and $g \geq 0$. The null hypersurface $f=0$ has been interpreted as a gravitational wave front with a background space-time in the region $f<0, f+g\rangle 0$. For a complete solution, it is also necessary to determine an extension of the solution into the region $g<0, f+g>0$ for which $\zeta<-1$. An extension into this region having a finite number of continuous derivatives can be achieved for the explicit solutions given above for integer $n$ simply by replacing $\arccos \zeta$ by $\pi+\operatorname{arccosh}(-\zeta)$ and $\sqrt{1-\zeta^{2}}$ by $-\sqrt{\zeta^{2}-1}$

Apart from the weak singularities (in the sense of analytical functions) representing the gravitational wavefronts, it is important to notice that the solutions constructed in this way for the vacuum Kasner background and the spatially flat and open FRW stiff fluid backgrounds are regular everywhere except at the initial singularity $t=0$. The significance of this will be noted later. At this stage, it may simply be noted that the gravitational waves may be considered to originate in the initial singularity.

\section{WAVE INTERACTIONS}

In order to consider the collision and subsequent interaction of such waves, it is first convenient to shift the wave front. It can be seen that the solution

$$
V_{\mathrm{II}}=\sum_{n=1}^{\infty} a_{n} t^{n} H_{n}\left(\zeta_{2}\right)
$$

where

$$
\zeta_{2}=\frac{2 c+z}{t}=\frac{2 c-f+g}{f+g}
$$

represents a gravitational wave with wavefront $f=c$. Similarly, the solution

$$
V_{\mathrm{III}}=\sum_{n=1}^{\infty} b_{n} t^{n} H_{n}\left(\zeta_{3}\right)
$$

where 


$$
\zeta_{3}=\frac{2 c-z}{t}=\frac{2 c+f-g}{f+g}
$$

represents a gravitational wave with wave front $g=c$ propagating in the opposite direction. The two sequences of constants $a_{n}$ and $b_{n}$ can be used to represent arbitrary profiles of the two waves.

For some positive constant $c$, these solutions may be taken to represent two approaching gravitational waves in the regions II $(f+g>0, f \geq c, g<c)$ and III $(f+g>0$, $g \geq c, f<c)$, respectively. The background ahead of each wave is the region $\mathrm{I}(f+g>0, f<c, g<c)$. These two waves collide when $t=2 c$, and the region IV ( $f \geq c$, $g \geq c)$ is the interaction region following the collision. It is assumed that an initial singularity occurs when $t=0$.

When the background region $I$ is the spatially flat or open FRW stiff fluid model or a type- $D$ Kasner spacetime in which $V=0$, the above solutions form a characteristic initial value problem for the collision of gravitational waves in these backgrounds. The two waves approach each other from opposite spatial directions relative to the fluid. It can then be seen that the unique solution representing the interaction region IV is given by

$$
V_{\mathrm{IV}}=\sum_{n=1}^{\infty} a_{n} t^{n} H_{n}\left(\zeta_{2}\right)+\sum_{n=1}^{\infty} b_{n} t^{n} H_{n}\left(\zeta_{3}\right)
$$

In this case it is more difficult to integrate Eqs. (4.4) to obtain $S_{\text {IV }}$. However, it is clear that the solution will be regular throughout the space-time.
It is also straightforward to obtain an exact solution for colliding gravitational waves in a vacuum Kasner background by including the expression $V_{\mathrm{I}}=a_{0} \ln t$ in all four regions.

It may now be recalled that the solutions constructed as described above for the vacuum Kasner background and the spatially flat and open FRW stiff fluid backgrounds are regular everywhere except at the initial singularity $t=0$. It is therefore concluded that interacting gravitational waves of the type represented here do not introduce future curvature singularities into the spacetime. This is in marked contrast to the case of the interaction of nonexpanding gravitational waves in a flat background. Here it has been shown that even a class of arbitrarily strong gravitational waves originating in the initial singularity are not sufficient to halt and reverse the expansion of the background space-time. Of course it is possible that very strong gravitational waves generated subsequent to the big bang may well introduce future singularities. However, this situation is well beyond the scope of this paper.

\section{ACKNOWLEDGMENTS}

The work of G.A.A. was supported, in part, by the Russian Foundation for Fundamental Research Grant No. 94-02-03678 and by Grant No. J1C100 from the International Science Foundation and Russian Government and by INTAS Grant No. 93-3262.
[1] J. B. Griffiths, Colliding Plane Waves in General Relativity (Oxford University Press, Oxford, England, 1991).

[2] V. A. Belinskii and V. E. Zakharov, Zh. Eksp. Teor. Fiz. 75, 1955 (1978) [Sov. Phys. JETP 48, 985 (1978)].

[3] V. A. Belinskii, Zh. Eksp. Teor. Fiz. 77, 1239 (1979) [Sov. Phys. JETP 50, 623 (1979)].

[4] E. Verdaguer, Phys. Rep. 229, 1 (1993).

[5] M. Carmeli, Ch. Charach, and S. Malin, Phys. Rep. 76, 79 (1981).

[6] J. Centrella and R. A. Matzner, Phys. Rev. D 25, 930 (1982).
[7] J. B. Griffiths, Class. Quantum Grav. 10, 975 (1993).

[8] J. B. Griffiths, J. Math. Phys. 34, 4064 (1993).

[9] J. Bičák and J. B. Griffiths, Phys. Rev. D 49, 900 (1994).

[10] A. Feinstein and J. B. Griffiths, Class. Quantum Grav. 11, L109 (1994).

[11] J. Wainwright, W. C. W. Ince, and B. J. Marshman, Gen. Relativ. Gravit. 10, 259 (1979).

[12] R. Tabensky and A. H. Taub, Commun. Math. Phys. 29, 61 (1973).

[13] A. Krasiński, Physics in an Inhomogeneous Universe (unpublished). 